\title{
The Prevalence of Suicidal Ideation among People Living With HIV and Aids Attending Art Clinic at Adult Centre of Excellence University Teaching Hospital, Lusaka
}

\author{
Monica M Mwenya ${ }^{1 *}$, Lottie Hachaambwa ${ }^{2}$, Ravi Paul ${ }^{3}$ and Joyce Ncheka \\ ${ }^{1}$ Department of Psychiatry, University of Zambia, Zambia \\ ${ }^{2}$ University Teaching Hospital, Lusaka and University of Maryland School of Medicine, Zambia \\ ${ }^{3}$ Department of Psychiatry, University of Zambia, Zambia
}

${ }^{4}$ Department of Psychiatry, University of Zambia, Zambia

Received: 漹 April 10, 2018; Published: 制 May 04, 2018

*Corresponding author: Monica Mpundu Mwenya, University of Zambia, School of Medicine, Department of Psychiatry, Lusaka, Zambia

\begin{abstract}
Background: Suicidal ideation has long been associated with HIV infected populations worldwide. It has been found that HIV does not only attack the immune system of an individual but also the nervous system leading to psychological dysfunction of an individual. Objective: To establish the prevalence of suicidal ideation among people living with HIV and AIDS. Method: A cross sectional quantitative design was adopted. Systematic random sampling method was used to select the sample. The total sample comprised of 280 participants. A social demographic questionnaire and Suicidal Risk Screening Scale (SRSS) were used to collect data. Results: The study findings from the SRSS test revealed that $(n=193,69 \%)$ of the participants had lower suicide risk while $(n=87,31 \%)$ fell into the higher suicide risk category. The study therefore showed that the prevalence of suicidal ideation was $31 \%$. Conclusion: Suicidal ideation was prevalent among people living with HIV and Aids.
\end{abstract}

Keywords: Suicidal ideation; Human immunodeficiency virus; Psychological dysfunction.

\section{Introduction}

IHuman Immunodeficiency Virus (HIV) was first reported in 1981 and has since become a major worldwide epidemic. HIV attacks the immune system of the body and causes the Acquired Immune Deficiency Syndrome (AIDS) [1]. When an individual has HIV the body becomes susceptible to diseases since the immune system has been reduced and can neither fight infections nor protect the person from diseases. This condition tends to trigger different psychological challenges among the people who are infected [2] found that since 1990, 271 or nearly $2 \%$ of approximately 14,000 people living with HIV who died in the United Kingdom had taken their own lives, and the proportion of deaths due to suicide had increased in the period since effective HIV treatment became available. It is generally believed that non-adherence to treatment is an expression of suicidal thoughts. The evidence from literature reveals that those who make suicidal attempts have seriously thought about doing so earlier. Sub-Saharan Africa has one of the highest global prevalence rates of HIV and AIDS. There are an estimated 24.7 million (23.5-26.1 million) People Living with HIV and AIDS (PLWHA) in sub-Saharan Africa [3]. The infection is more prevalent in Africa among developing countries and South Africa is considered to be one of the world's worst affected by HIV and AIDS and about 5.7 million people are affected and one in three pregnant women are living with HIV and AIDS [4].

Zambia being a developing country has not been spared from prevalence of HIV, and the adult HIV prevalence rate stood at $14.3 \%$ in 2007 [5] and is still high at 12.5\% [3]. Furthermore, the knowledge of HIV status has major implications for individuals who are positive with the infection. A study conducted in South Africa among PLWHA revealed that 24\% who were tested had suicidal ideation [6]. In South Africa, many suicides and attempted 
suicides go unreported, but available statistics are alarming, with prevalence rate of between $17-25$ per 100,000 of the population and an attempted suicide ratio of about 1:20 [7]. Suicide accounts for about $9.5 \%$ of non-natural deaths in young people and $11 \%$ in adults in the country, with the average age of suicide being 35 years and for suicide attempts 20-29 years followed by the 1019-year age group. Consequently suicidal ideation, attempts, and completions remain alarmingly common among people living with HIV and AIDS (PLWHA), despite a recorded decline in suicide rates since the advent of Highly Active Antiretroviral Therapy (HAART) in the 1990s to levels comparable with those of other chronic disease afflicted populations [7].

\section{Statement of the problem}

Suicidal ideation is one of the public concerns among people living with HIV and AIDS. Suicidal thinking may occur among people living with HIV and AIDS, triggering harmful impacts on the quality of life, treatment adherence, and disease progression [8]. The HIV and AIDS infection attacks the immune and nervous systems leading to psychological dysfunction. Prolonged conditions of HIV and AIDS subject people to suicidal ideation and attempted suicide and in some cases lives have been lost [8] and [7]. Suicidal ideations are significantly common among persons living with HIV and AIDS compared to non-infected controls and have been reported in most cases to be associated with psychiatric disorder [9]. Yet the psychosocial factors contributing to these psychiatric disorders remain unreported, particularly in the developing country context and mostly, in sub-Saharan African countries [10]. In Zambia, there has been no evidence of research publications on the prevalence of suicidal ideation among people living with HIV and AIDS. Therefore, this research sought to investigate the prevalence of suicidal ideation among people living with HIV and AIDS.

\section{Objective of the study}

To investigate the prevalence of suicidal ideation among people living with HIV and AIDS attending ART clinic at Adult Centre of Excellence at UTH in Lusaka.

\section{Literature Review}

\section{Introduction}

High rates of suicide and accidental or violent death have also been described in HIV infected populations including in those receiving effective ART [11]. The extent to which HIV infection is also associated with increased risk of suicidal ideation is not well documented. Hence, most HIV-related studies focus on suicide as an endpoint [12]. As such this has resulted in less studies focussing on the aspect of prevalence suicidal ideation. According to $[13,14]$. Suicidal thought involves a range of suicidal behaviours, which sometimes may be fatal or non-fatal. In a study of British private households, some researchers found differences in the risk pattern of suicidal thoughts compared to completed suicide [15]. In that study the incidence of suicidal thoughts was seen to be over 200 times greater than the incidence of suicide. Therefore, from the literature given, people living with HIV and AIDS tend to be more subjected to negative thoughts which lead to poor quality of life with suicidal thoughts.

\section{Neurological changes}

The researchers [16] stated that the nervous system is the worst impaired system in HIV condition after the immune systems. This dysfunction has an effect on the psychological aspect of PLWHA which makes them vulnerable to self-harming. Several studies have reported that, HIV infection has an effect on neuropsychological functioning ranging from mild to severe [17]. Hence, neurological changes are some of the causes of suicidal ideation as these substrates also affect the ability to initiate action or thought and regulate mood, and they promote persistent perseveration dysfunctions $[18,19]$. These neurological changes may bring about negative thoughts towards physical appearances and psychological dysfunctions.

In addition, the prevalence of self-harm or suicidal ideation will tend to persist in the condition of repeated suicidal thinking among individuals living with HIV and AIDS. Therefore, Emotional difficulties along with the tendency for perseveration may influence the ability to think about problems while impairing the ability to think logically. In fact, according to [20] adults who have a ruminative personality style are more likely to contemplate suicide. These adults are likely to become stressed up on negative issues as they go into deeper thoughts with anxiety. Consequently, more severe side effects of ART, detectable HIV viral load, and a critically low T-helper CD4 cell counts may also be related to suicidal ideation. As individuals have low CD4 cell counts, they are subjected to poor health conditions in which contracting of multiple infections cannot be prevented and suicidal ideation is likely to persist on their psychological well-being [21].

\section{Suicidal Ideation and its Prevalence}

Suicidal thinking may occur among individuals with HIV and AIDS, triggering profound harmful impacts on the quality of life, treatment adherence, disease progression, and mortality [22]. Hence, suicidal ideation has been commonly found in some studies as psychiatric disorders. The prevalence of suicidal ideation in another study revealed that such disorders may arise as a direct result of HIV neuro- invasion or psychosocial stressors, or due to complications of ART $[23,24]$. As such HIV has increased frequency and severity of both suicidal ideation and thoughts of death among adults. The risk of suicide is especially high for patients who are at serious points in the course of HIV infection. Suicidal ideation has been proved to be found in a study of adults living with HIV and AIDS, in which [25] reported that those who self-rated their medication side effects and HIV related symptoms as being severe were more likely to report suicidal ideation. The prevalence of such high rates of suicidal ideation indicates that the stressors associated with HIV are severe enough to have an impact on the quality of life in this population even though a person is on ART. These high rates during all stages of HIV suggest that other factors are involved. 


\section{Prevalence of Suicidal Ideation among people living with HIV and AIDS}

In a study by $[25,26]$ it was found that that the presence of suicidal ideation increases the risk of suicidal attempt and completed suicide among PLWHA. Studies also suggest that patients' risk for suicide may be greater soon after testing positive for HIV than later on, as after some time has passed they begin to adjust to living with the infection [27]. However, thoughts of suicide may reduce as people adjust to their HIV positive status; though there may be resurgence in suicide risk as HIV-related disease advances, particularly with the development of AIDS-related symptoms and illnesses. In addition, in Switzerland a study by the Swiss HIV Cohort Study demonstrated where rates of suicide decreased substantially in the ART era compared to the pre ART era but still remain well above that observed in the general population [28]. Therefore, the rates of suicide risks in HIV infected patients may be higher than in population with other chronic medical illnesses like cancer.

A study in Australia, revealed that People who experience suicidal ideation and those who make suicide plans are at increased risk of suicidal attempts, and people who experience all forms of suicidal thoughts and behaviours are at greater risk of completed suicide [29]. Adult people living with HIV tend to look down on themselves and their minds contemplate death as a solution to their predicament. Furthermore, results have shown an estimated $13.3 \%$ of community dwelling adults in Australia experience suicidal ideation during their lifetime with $3.2 \%$ attempting suicide highlighting an important public health problem [30]. In other studies, done in India, it has been shown that Suicidal ideation is a recurrent theme seen in the HIV literature and often associated with major depression and hopelessness [31,32]. This pinpoints the prevalence of suicidal thoughts due to psychological dysfunctions among PLWHA.

The few African studies on suicidal thoughts in HIV and AIDS have reported the following prevalence rates: $12.4 \%$ for suicidal ideation among patients attending a specialized HIV and AIDS clinic in pre-ART Uganda; $17.1 \%$ among adolescents living with HIV in pre-ART Uganda; $13 \%$ for current suicidal ideation among patients attending a specialised HIV and AIDS clinic in post-ART Uganda [33]. From the evidence given in Uganda, it is obvious that suicidal thoughts do not exclude adolescents who are in the transition to adulthood and experiencing health impairments in their life time.

In Nigeria, researchers while presenting the prevalence of suicide attempts in individuals living with HIV and AIDS, [34] revealed the prevalence of attempted self harm but did not report on the determinants of suicidal thoughts. Moreover, studies in South Africa showed that suicidal ideation was at $12 \%$ amongst adolescents and $9.1 \%$ amongst adults in the general population [35]. Hence, suicidal thought is found even among adolescents more especially during the transition into adulthood as they go through a lot of physiological changes and face psychological problems, the prevalence has been seen to be higher in adolescents than adults as per the previous studies in South Africa.
A study in Zimbabwe was conducted with the objective of examining the prevalence of HIV and AIDS infection in neuropsychiatric disorders, psychiatric symptoms or signs. The study demonstrated that among patients living with HIV and AIDS who were depressed, showed significant symptoms or signs such as lassitude and pessimistic suicidal thoughts [36]. Most of psychosocial dysfunctions are manifested with their clinical symptoms and behaviour which are clear determinants of suicidal ideation. Psychiatric symptoms among persons living with HIV and AIDS have been studied in Zimbabwe and have revealed the prevalence of suicidal thoughts but not among the HIV seronegative individuals.

\section{Methodology}

The study used a cross sectional quantitative method. The study population were individuals living with HIV and AIDS attending ART clinic at Adult Centre of Excellence, UTH in Lusaka. The study targeted individuals living with HIV and AIDS aged between 18 to 60 years. The study sample consisted of 280 participants with 115 (41.1\%) men and 165 (58.9\%) women. A systematic random sampling technique using intervals was used to give every element equal chance of being selected. The researcher picked every third person from the last one. On average 70 to 80 patients attended the clinic per day. The researcher was only able to interview between six (6) and ten (10) participants per day and only those who gave consent and met the inclusion criteria. The research used a questionnaire to gather demographic information that included items such as age, level of education, employment status, income level, gender, marital status, duration of living with HIV status.

The third instrument used was Suicidal Risk Screening Scale (SRSS) to identify those with suicidal ideation, the scoring was $0=$ True, then $1=$ False, total score 14 . The SRSS indicated, Lower suicide risk: 3 and possible higher risk: 4 or greater for suicidal ideation (Figure 1) shows results for the prevalence of suicidal ideation. Results show that $(n=87,31 \%$ ) of the respondents fell into the category of possible higher suicidal ideation risk category while ( $n=193,69 \%$ ) fell into the lower suicidal ideation risk category. The study therefore showed that the prevalence rate of suicidal ideation in the population of people living with HIV and AIDS was $31 \%$ (Table 1).

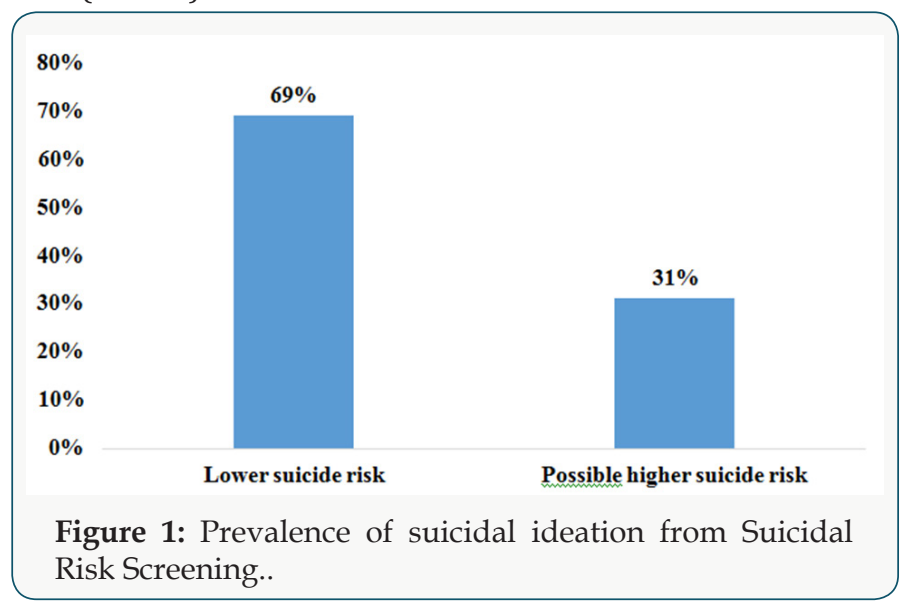


Table 1: Social Demographic Characteristics of the Sample Frequency table showing characteristics of sample $(\mathrm{N}=280)$.

\begin{tabular}{|c|c|c|c|c|}
\hline & & Count & Percent & $95 \% \mathrm{CI}$ \\
\hline \multirow[t]{2}{*}{ Gender } & Male & 115 & $41.1 \%$ & $35.4-47.0$ \\
\hline & Female & 165 & $58.9 \%$ & $53.0-64.6$ \\
\hline \multirow[t]{7}{*}{ Age } & $18-24$ & 76 & $27.1 \%$ & 22.2-32.7 \\
\hline & $25-30$ & 17 & $6.1 \%$ & $3.8-9.6$ \\
\hline & $31-36$ & 43 & $15.4 \%$ & $11.6-20.1$ \\
\hline & $37-42$ & 49 & $17.5 \%$ & $13.5-22.4$ \\
\hline & $43-48$ & 38 & $13.6 \%$ & $10.0-18.1$ \\
\hline & $49-54$ & 37 & $13.2 \%$ & $9.7-17.7$ \\
\hline & $55-60$ & 20 & $7.1 \%$ & $4.6-10.8$ \\
\hline \multirow[t]{4}{*}{ Marital status } & Single & 119 & $42.5 \%$ & $36.8-48.4$ \\
\hline & Married & 122 & $43.6 \%$ & $37.8-49.5$ \\
\hline & Widowed & 22 & $7.9 \%$ & $5.2-11.7$ \\
\hline & Divorced & 17 & $6.1 \%$ & $3.8-9.6$ \\
\hline \multirow[t]{6}{*}{ Education } & Grade 7 & 15 & $5.4 \%$ & $3.2-8.7$ \\
\hline & Grade 9 & 61 & $21.8 \%$ & $17.3-27.0$ \\
\hline & Grade 12 & 101 & $36.1 \%$ & $30.6-41.9$ \\
\hline & Diploma & 47 & $16.8 \%$ & $12.8-21.7$ \\
\hline & Degree & 27 & $9.6 \%$ & $6.7-13.7$ \\
\hline & Other & 29 & $10.4 \%$ & $7.3-14.5$ \\
\hline \multirow[t]{4}{*}{ Employment } & Unemployed & 96 & $34.3 \%$ & $28.9-40.1$ \\
\hline & Self employed & 83 & 29.6 & $24.6-35.3$ \\
\hline & Unemployed & 90 & 32.1 & $26.9-37.9$ \\
\hline & Retired & 11 & 3.9 & $2.2-7.0$ \\
\hline \multirow[t]{4}{*}{ Income } & Below K10, 000 & 168 & $60.0 \%$ & $54.1-65.6$ \\
\hline & $\mathrm{K} 10,000-\mathrm{K} 20,000$ & 44 & $15.7 \%$ & $11.9-20.5$ \\
\hline & $\mathrm{K} 20,000-\mathrm{K} 30,000$ & 24 & $8.6 \%$ & $5.8-12.5$ \\
\hline & $\mathrm{K} 30,000$ and above & 44 & $15.7 \%$ & $11.9-20.5$ \\
\hline \multirow[t]{4}{*}{ Duration of HIV status } & Less than 6 months & 25 & $8.9 \%$ & $6.1-12.9$ \\
\hline & $6-12$ months & 22 & $7.9 \%$ & $5.2-11.7$ \\
\hline & 1-3 years & 52 & $18.6 \%$ & $14.4-3.6$ \\
\hline & More than 3 years & 181 & $64.6 \%$ & $58.8-70.1$ \\
\hline
\end{tabular}

\section{Discussion of Findings}

The findings of the study showed the importance of the psychological tests that usually remain routinely unused at baseline level by medical professionals in health institutions especially in developing countries like Zambia. The tests can go a long way in bringing to the fore the vulnerability of HIV patients to the risk of suicidal ideation or risk of committing suicides. Prevalence of Suicidal Ideation among people living with HIV and AIDS attending Adult Clinic Centre of Excellence. The findings from the Suicidal Screening Risk Scale (SRSS) showed that $31 \%$ of the respondents fell in the higher suicidal ideation risk category. This is in line with the study by who found that individuals who self-rated their medication side effects and HIV related symptoms as being severe were more likely to be susceptible to suicidal ideation. The findings were also in line with the findings of in South Africa whose study found that $25.4 \%$ of respondents had suicidal thoughts, while $15.6 \%$ had plans to commit suicide.

Another study that is in line with the findings of this study is one by [37] who found that depression among women attending antenatal clinic was between $30 \%$ to $47 \%$. In South Africa, found that $12 \%$ of respondents with suicidal ideation were among adolescents and $9.1 \%$ among adults in the general population. Hence, suicidal thoughts is found even among adolescents more especially during the transition into adulthood as they go through a lot of physiological changes and face psychological problems, the prevalence has been seen to be higher in adolescents than adults as per previous studies in South Africa. In addition, the above results are in line with the findings on the prevalence of suicidal ideation 
in Uganda. According to $10 \%$ of the respondents on suicidality in HIV and AIDS individuals in Entebbe Uganda had met the criteria of suicidal ideation. In addition, in Uganda also found that there were similarities in his findings as he found, $12.4 \%$ suicidal ideation among patients attending a specialised HIV and AIDS Pre-Art clinic, $17.1 \%$ among adolescents at the same clinic, and $13 \%$ of patients attending a Post Art Clinic.

\section{Conclusion}

In conclusion, the study has shown that suicidal ideation is prevalent among people living with HIV and AIDS though it has been overlooked in Zambia as a health concern. In this study, the suicidal ideation assessment revealed that $31 \%$ of the respondents showed suicidal ideation. The study has also showed that the SRSS can be a valuable tool for screening suicidal ideation in people living with HIV and AIDS.

\section{References}

1. Chiboola H (2003) HIV/AIDS counseling: A Handbook. Zambia Counseling Council (ZCC). Lusaka: Associated Printers.

2. United Nations Programme for AIDS (2014) 'The Gap Report'. UNAIDS.

3. Schlebusch L, Vawda N (2010) HIV-infection as a self-reported risk factor for attempted suicide in South Africa. Afr J Psychiatry 13(4): 280283.

4. Zambia Demographic Health Survey (2009) Central Statistical Office (CSO) Ministry of Health, Tropical Diseases Research Centre, University of Zambia \& Macro International Inc.

5. Govender L, Schlebush (2013) A Suicide risk screening scale for HIV infected persons in the immediate post diagnosis period 14(2).

6. Schlebusch L, Burrows S (2009) Suicide Attempts in Africa In: Wasserman D, Wassermann, editors; Oxford Textbook of Suicidology and Suicide Prevention, A Global Perspective. Oxford University Press; Oxford: UK pp. 105-108.

7. Shirey KG (2013) Suicide and HIV. Mental Health Practitioner's Guide to HIV. New York, UK 405-407.

8. Sherr L, Clucas C, Harding R, Sibley E, et al. (2011) HIV and depression, a systematic review of interventions. Psychol Health Med 16(8): 493-527.

9. Kelly B, Raphael B, Judd F, Perdices M, Kernutt G, et al. (1998) Suicidal ideation, suicide attempts, and HIV infection. Psychosomatics 39(5): 405-415.

10. Amiya RM, Poudel KC, Poudel-Tandukar K, Pandey BD, Jimba M (2014) Perceived Family Support, Depression and Suicidal Ideation among People Living with HIV/AIDS. Kathmandu Valley: Nepal.

11. Rice BD, Smith RD, Delpech VC (2010) HIV infection and suicide in the era of HAART in England, Wales and Northern Ireland. AIDS 24(11): 1795-1717.

12. Catalan J, Harding R, Sibley E, Clucas C, Croome N (2011) HIV infection and mental health: suicidal behavior systematic review. Psychol Health Med 16(5): 588-611.

13. Bech P, Awata S (2009) Measurement of suicidal behavior with psychometric scales. In: Wasserman D, Wasserman C, Oxford Textbook of Suicidology and Suicide Prevention. A Global Perspective. Oxford: Oxford University Press, UK 305-311.

14. Bantjies J, Van Ommen C (2008) The Development and Utilization of A Suicide Risk Assessment Interview Schedule. S Afr J Psychol 38(2): 391- 411.
15. Gunnell D, Harbord R, Singleton N, Jenkins R, Lewis G (2004) Factors influenced the development andamelioration of suicidal thoughts in the general population. Cohort study. Br Psychiatry 185: 385-393.

16. Williams KC, Hickey WF (2002) Central nervous system damage monocytes and macrophages and neurological disorders in AIDS. Annual Review of Neurosciences 25: 537-562.

17. Heaton RK, Marcotte TD, Rivera-Mindt M, Sadek J, Moore DJ, et al. The Impact of HIV-Associated Neuropsychological Impairment on Everyday Functioning. Psychology Faculty Publications. Paper 15.

18. Koutsilieri E, Sopper S, Scheller C, Ter Meulen V, Riederer P (2002) Parkinsonism in HIV dementia. Journal of Neural Transmission 109(56): 767-775.

19. Vance DE, Robison FP (2004) Reconciling successful aging with HIV: A biopsychosocial overview. Journal of HIV/AIDS and Social/Sciences 3: 59-78.

20. Fair weather AK, Anstey KJ, Rodgers B, Jorm AF, Christensen H (2007) Age and gender differences among Australian suicide ideators: Prevalence and correlates. The Journal of Nervous and Mental Disease 195(2): 130-136.

21. Sherr L, Lampe F, Fisher M, Arthur G, Anderson J, et al. (2008) Suicidal ideation in UK HIV clinic attendees. AIDS 22(13): 1651-1658.

22. Spudich S, Gonzalex-Scarano F (2012) HIV-1-related central nervous system disease: Current issues in pathogenesis, diagnosis and treatment. Cold Spring Harb Perspect Med 2(6): 1101.

23. Minager A, Commins D, Alexander JS, Hoque R, Singer EJ, et al (2008) NeuroAids: Characteristics and diagnosis of the neurological complications of AIDS. Mol Diagn Ther 12(1): 25-43.

24. Carrico AW, Johnson MO, Morin SF, Remien RH, Charlebois ED, et al. (2007) NIMH Health Living Project Team: Correlates of suicidal ideation among HIV-positive persons. AIDS 21(9): 1199-1203.

25. Lawrence ST, Willig IH, Crane HM, Ye J, Aban I, et al. (2010) Routine, self-administered, touch screen, computer-based suicidal ideation assessment linked to automated response team notification in an HIV Primary Care setting Clinical infectious diseases 50(8): 1165-1173.

26. Shittu RO, Alabi MK, Odeigah LO, Sanni MA, Issa BA, et al. (2014) Suicidal Ideation among Depressed People Living with HIV and AIDS. Nigeria, West Africa 3(3): 262-270.

27. Dannenberg AL, McNeil JG, Brundage JF (1996) Suicide and HIV infection. Mortality follow-up of 4147 HIV-sero positive military service applicants. JAMA 276(21): 1743-1746.

28. Keiser O, Spoerri A, Brinkhof MW, Hasse B, Gayet-Ageron A, Tissot F, et al. (2010) Suicide in HIV-infected individuals and the general population in Switzerland. American Journal of Psychiatry 167(2):143-150.

29. Slade T, Johnston A, Teesson M, Whiteford H, Burges P, et al. (2009) The Mental Health of Australians 2. Report on the 2007 National Survey Mental and Wellbeing. Canberra: Department of Health and Ageing.

30. Johnston AK, Pirks JE, Burgess PM (2009) Suicidal thoughts and behaviors among Australia adults: findings from the 2007 National Survery of Mental Health and Wellbeing. Aust N Z J Psychiatry 43(7): 635-643.

31. Chandra PS, Desai G, Ranjan S (2005) HIV \& psychiatric disorders Indian Journal of Medical Research 121(4): 451-467.

32. Santosh P (2004) Clinical predictors of suicidal ideation in HIV seropositive population. MD thesis. Bangalore: National Institute of Mental Health and Neurosciences.

33. Gali BM, Naaya HU, Adamu S (2004) Suicide Attempts in HIV/AIDS patients: Report of Two Cases Presenting with Penetrating Abdominal Injuries. Nigerian Journal of Medicine 13: 407-409. 
34. Mashego AB, Madu SN (2009) Suicide-related behaviors among secondary school adolescents in the Welkom and Bethlehem areas of the Free State province (South Africa). S Afr J Psychol 39: 489-506.

35. Sebit MB, Chandiwa SK, Latif AS (2002) Neuropsychiatric aspects of HIV disease progression: Impact or traditional herbs on adult patients in
Zimbabwe. Progress in Neuropsychopharcology and Biology psychiatry 26: 451-456.

36. Manikkam L, Burns JK (2012) Antenatal depression and its risk factors: An urban prevalence study in KwaZulu-Natal. South African Medical Journal 102(12): 940-944.

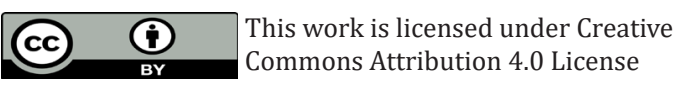

To Submit Your Article Click Here:

Submit Article

DOI: $10.32474 / 0 J N B D .2018 .01 .000108$

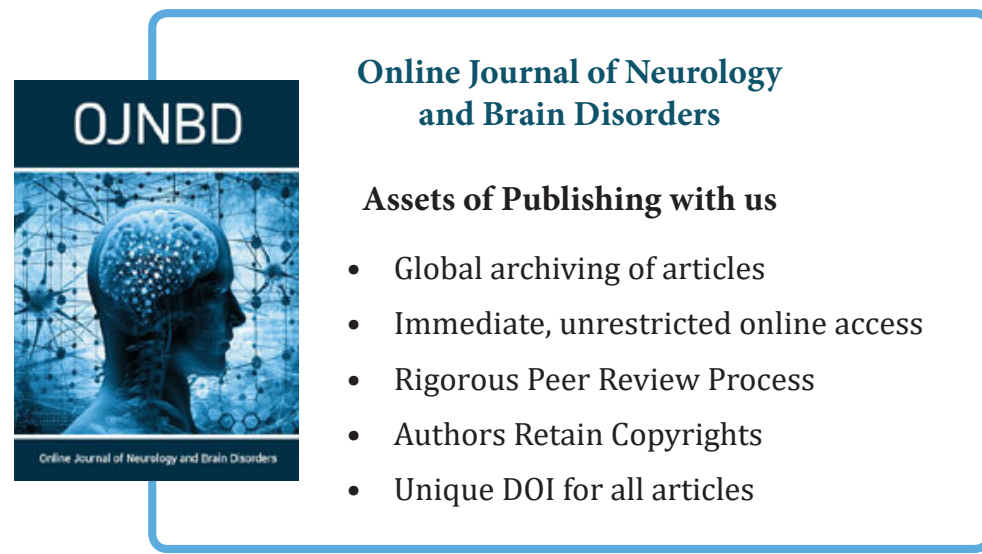

